\title{
Ultradünner Stent macht Klassenprimus Konkurrenz
}

\author{
Der Xience-Stent - der Big Player unter den medikamentenbeschichteten \\ Stents - bekommt ernstzunehmende Konkurrenz. Mit einem ultradünnen \\ Stent ließen sich nun erstmals bessere Ergebnisse erzielen.
}

$\mathrm{D}$ er Xience-Stent muss sich erstmals in einem Direktvergleich mit einem Konkurrenten geschlagen geben. Nach Implantation des ultradünnen OrsiroStents mit einer biodegradierbaren Polymerbeschichtung war die Rate an Zielläsions-Versagen geringer als mit dem derzeitigen Marktführer.

Mit diesen Ergebnissen könnte sich ein neuer Standard für den Vergleich medikamentenbeschichteter Stents („drug eluting stents“, DES) etablieren, äußerte sich der Studienleiter Dr. David Kandzari auf einer ESC-Session zu den neuen Daten.

\section{Vorteile: dünne Streben,} abbaubare Polymer-Beschichtung Das Besondere an dem Orsiro-Stent sind seine ultradünnen Stentstreben $(60 \mu \mathrm{m})$ und die bioresorbierbare Polymer-Matrix, die mit Sirolimus beschichtet ist. Im Gegensatz dazu weist der Xience-Stent eine permanente Everolimus-freisetzende Polymerbeschichtung und eine Strebendicke von $81 \mu \mathrm{m}$ auf.
Entwickelt wurden die neuen DES mit biodegradierbarer Polymerbeschichtung in der Hoffnung, damit das Risiko für späte und sehr späte Stentthrombosen weiter reduzieren zu können. Denn nach Auflösung der Beschichtung ist der Stimulus für Entzündungsprozesse gering und Hypersensitivitätsreaktionen werden vermieden.

\section{Bisher nur Nichtunterlegenheit gezeigt}

Doch bisher ist es für keinen solchen DES gelungen, eine statistische Überlegenheit gegenüber dem aktuellen Standard Xience-Stent zu zeigen. Einzig die Nichtunterlegenheit konnte belegt werden, berichtete Kandzari.

Mit der Vormachtstellung des XienceStents könnte es nun aber vorbei sein. Die randomisierte kontrollierte BIOFLOW V-Studie mit 1.334 KHK-Patienten war eigentlich darauf angelegt, eine Nichtunterlegenheit des Orsiro-Stent gegenüber dem Xience-Stent zu zeigen. Dieser Nachweis gelang dem neuen DES.

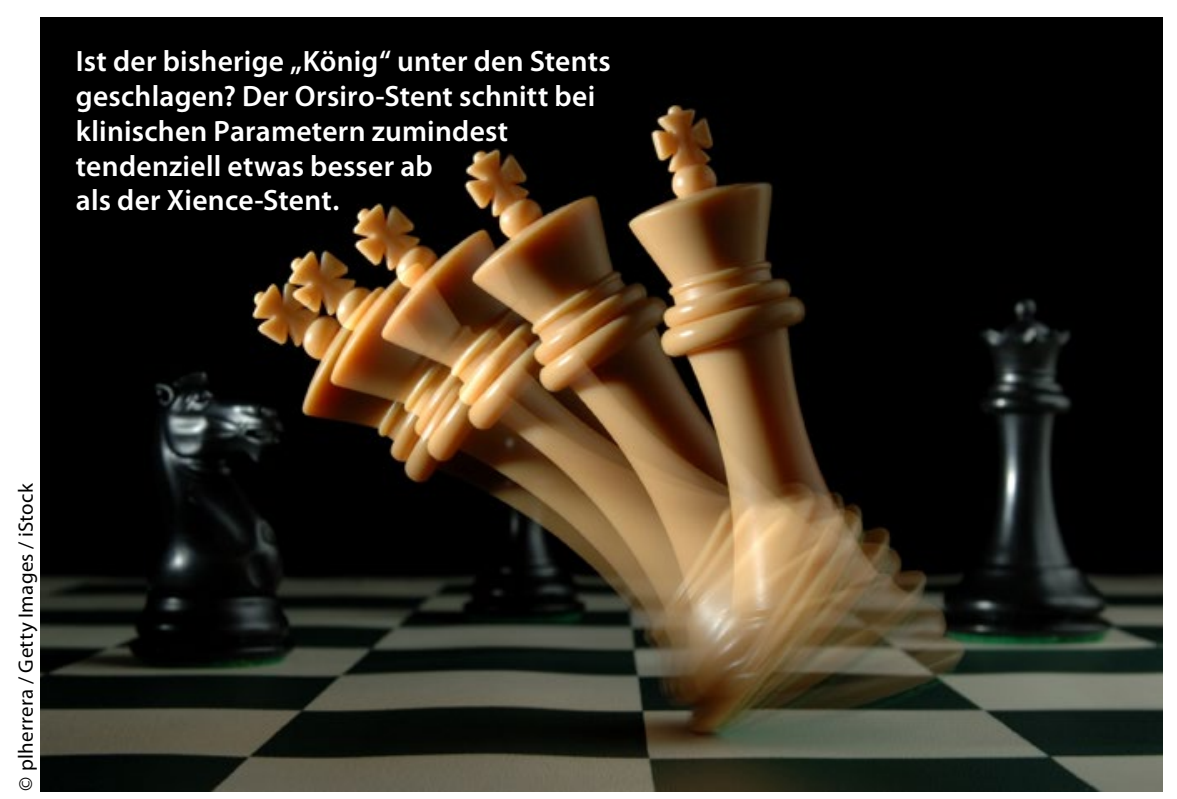

Orsiro-Stent nun überlegen

So kam es bei den 884 Patienten, die den Orsiro-Stent implantiert bekommen haben, nach 12 Monaten in 6,2\% der Fälle $\mathrm{zu}$ einem Zielläsions-Versagen (,target lesion failure“, TLR), definiert als kardialer Tod, Myokardinfarkt im Zielgefäß oder klinisch notwendige erneute Revaskularisation im Zielgefäß. Nach Implantation des Xience-Stents trat dieser primäre Endpunkt bei 9,6\% der damit behandelten Patienten $(\mathrm{n}=450)$ ein $(\mathrm{p}=0,040)$. Dieser Unterschied kam vor allem durch die deutlich geringere Rate an Myokardinfarkten im Zielgefäß zustande $(4,7$ vs. $8,3 \%$; $p=0,016)$.

Die sich hier abzeichneten Überlegenheit konnte dann in einer gepoolten Auswertung von Daten der BIOFLOW V, II und IV-Studien mit 2.208 Patienten statistisch nachgewiesen werden. Diese Analyse ergab eine TLR-Rate von 6,3\% mit dem Orsiro-Stent vs. 8,9\% mit dem Xience-Stent.

\section{Weniger Herzinfarkte - vor allem in der frühen Phase}

Auch bei klinischen Parametern schnitt der Orsiro-Stent tendenziell etwas besser ab. Nach 30 Tagen waren in der damit behandelten Gruppe weniger Herzinfarkte aufgetreten (4,3 vs. 6,9\%). Dieser Unterschied zeichnete sich schon früh in der periprozeduralen Phase $a b$, da vor allem die Rate an in der Klinik vorgekommenen Myokardinfarkten reduziert wurde (3,9\% vs. 6,7\%).

Stentthrombosen waren generell selten und deren Häufigkeit in beiden Behandlungsarmen vergleichbar. Einzig sehr späte Stentthrombosen jeglicher Art ( $\geq 30$ Tagen $\leq 1$ Jahr) kamen mit dem Orsiro-Stent signifikant seltener vor $(0,1$ vs. $0,9 \%)$.

Veronika Schlimpert

Quelle: Late-Breaking Science in $\mathrm{PCI} 1$ "BIOFLOW-V: A Prospective Randomized Multicenter Study to Assess the SaFety and Effectiveness of the Orsiro SiroLimus Eluting Coronary Stent System in the Treatment Of Subjects With up to Three De Novo or Restenotic Coronary Artery Lesions"; ESC-Kongress, 26.-30.8.2017, Barcelona

Kandzari DE et al. Ultrathin, bioresorbable polymer sirolimus-eluting stents versus thin, durable polymer everolimus-eluting stents in patients undergoing coronary revascularisation

(BIOFLOW V): a randomized trial. Lancet. 2017; https://doi.org/10.1016/S0140-6736(17)32249-3 